\title{
Epidemiology and outcome of COVID-19 among 260 confirmed cases admitted at Guder Hospital, Western Ethiopia, 2020: a descriptive study of the first ten months
}

\author{
Tesfaye Solomon ( $\square$ abdiikoo50@gmail.com ) \\ Ethiopian Public Health Institute https://orcid.org/0000-0003-0994-393X \\ Tufa Kolola \\ Department of Public Health, Ambo University, Ethiopia \\ Mekdes Tsegaye \\ Guder Hospital, Oromia Regional State, Ethiopia
}

\section{Research Article}

Keywords: Epidemiology, Outcome, COVID-19, Guder Hospital, Ethiopia

Posted Date: June 3rd, 2021

DOl: https://doi.org/10.21203/rs.3.rs-587354/v1

License: (c) (1) This work is licensed under a Creative Commons Attribution 4.0 International License.

Read Full License 


\section{Abstract}

Background: Coronavirus disease 19 (COVID-19) is a highly transmittable and pathogenic viral infection that emerged in Wuhan, China, and spread around the world. No evidence is available about the epidemiological, clinical features, and outcomes of admitted patients in the study area. This study aimed to provide insight into the epidemiological characteristics and outcome of confirmed COVID-19 infection among admitted cases at Guder Hospital, Oromia Regional State, Ethiopia.

Methods: In this descriptive study, all confirmed cases of COVID-19 admitted to Guder Hospital from May 16 to December 31, 2020, were included. Cases were confirmed by real-time PCR and analyzed using SPSS version 22. The data were collected from January 15-20, 2021.

Results: A total of 260 confirmed patients were admitted, including 180 men and 80 women. Of these cases, 184 (70.8\%) were asymptomatic at admission. The mean age of the patients was 35.5 [SD 15] years. Adults aged 15 and above years accounted for 254 (97.7\%). Meantime in the hospital was 14.3 [SD 3] days. On admission, the frequent symptom was cough (43[16.5\%]). The case fatality rate among the admitted cases was 12 (4.6\%). Of these deaths, seven (58.3\%) were females aged $45-59$ years, and eight had known comorbidity.

Conclusion: The COVID-19 infection is more likely to affect adult males and death is common in females with comorbidities. The disease remains largely asymptomatic among the admitted cases. A further analytical investigation is needed to explore the risk of mortality and its determinants among admitted cases with COVID-19 infection.

\section{Introduction}

Coronavirus disease 19 (COVID-19) which is caused by Severe Acute Respiratory Syndrome Coronavirus 2 (SARS-COV-2) is a highly transmittable and pathogenic viral infection that emerged in Wuhan, China in December 2019 and spread around the world[1, 2]. COVID-19 viruses are usually transmitted through respiratory droplets by direct contact with infected persons, or by contact with contaminated objects and surfaces[1].

The World Health Organization (WHO) has declared the COVID-19 outbreak as a public health emergency of international concern on January $30^{\text {th }}, 2020$ [3] and a pandemic on March 11 th, 2020 when the novel coronavirus total case count surpassed that for Severe Acute Respiratory Syndrome Coronavirus 1 (SARS-COV-1) which affected 8,096 people globally [4]. The coronavirus COVID-19 is affecting 218 countries and territories around the world and two international conveyances $[2,5]$.

The first case in Ethiopia was reported on March 13, 2020[6]. As of December 31, 2020, the total number of cases had risen to 124,264 cases, of which 112,096 have recovered. Among 114,019 closed cases that had an outcome, 112,096 (98.3\%) have recovered and 1,923(1.7\%) have sadly died[7]. On April 14, 2020, Ethiopia has declared a five-month State of Emergency to limit the spread of the Coronavirus[8]. 
People with COVID-19 have had a wide range of symptoms reported-ranging from mild symptoms to severe illness and may appear 2-14 days after exposure to the virus. Based on all 72,314 cases of COVID19 confirmed, suspected, and asymptomatic cases in China as of February 11, 2020, has found that $80.9 \%$ of infections are mild with flu-like symptoms and can recover at home while $13.8 \%$ are severe, developing severe diseases including pneumonia and shortness of breath. About $4.7 \%$ are critical and can include multi-organ failure finally which results in death[9].

Initially, the Federal Ministry of Health (FMOH) and Public Health Institute of Ethiopia (EPHI) recommended the management of all confirmed COVID-19 cases must be at the treatment center. Gradually, the concept of home-based isolation and care (HBIC) was considered since July 2020 due to the overflow of patients and limitation of resources at health facilities and the majority of the cases need no treatment. Currently, only moderate and severe COVID-19 patients are allowed to seek treatment at the treatment center where they get treatment for non-COVID-related morbidities and disease conditions[10].

A patient diagnosed with the COVID-19 can be discharged from the treatment center when the symptoms are subsisted, the body temperature remains at a normal range for at least three days, and two consecutive laboratory tests are negative[11].

The first laboratory-confirmed COVID-19 cases in West Shewa Zone were reported within a family on 16 May 2020 from Ginchi town, Dendi district, and admitted to Guder Hospital[12]. In West Shewa Zone of Oromia Regional State, 14,914 individuals were tested for COVID-19 during the period 13 March to 31 December 2020 of which 542 were confirmed cases and 19 deaths (3.5\%) in the zone[12].

No evidence is available about the epidemiological, clinical features, and outcome of admitted patients with COVID-19 in the study area. Therefore, further description of the hospitalized cases and their clinical outcomes are important to understand the current epidemiology of COVID-19 and help to set up our health facilities readiness accordingly. This study aims to investigate the epidemiology and clinical outcomes of COVID-19 among hospitalized cases in West Shewa Zone, the fourth most populous zone in Oromia Regional State, Ethiopia, during the early ten months of the outbreak.

\section{Methods And Materials}

\section{Study area and setting}

This study was conducted in Guder Hospital. Guder Hospital is one of the primary hospitals in West Shewa Zone which is located $126 \mathrm{~km}$ from the capital city of Ethiopia, Addis Ababa to the western part. According to the arrangements put in place by the Federal Government, all patients of confirmed COVID19 are admitted at a selected case treatment center (CTC). Guder Hospital is one of the selected CTC admitting those positive cases from the Western part of Oromia, Ethiopia.

\section{Study design and period}


A descriptive cross-sectional study was conducted retrospectively using secondary data of confirmed COVID-19 cases admitted to Guder Hospital from May 16, 2020, to December 31, 2020. The data were collected from January 15-20, 2021.

\section{Study variables}

Dependent variable: Outcome status of COVID-19 infection

Independent variables: The database includes socio-demographic variables, geographical location and time information, and clinical characteristics as variables

\section{Sample size calculation and sampling techniques}

All cases admitted to Guder Hospital who was diagnosed as having COVID-19 infection with RT-PCR diagnosis were included in this study from a database.

\section{Data collection instrument and techniques}

Data of confirmed COVID-19 cases admitted to Guder Hospital from May 16, 2020, to December 31, 2020, were collected from the database available at the hospital.

\section{Data quality assurance}

To ensure data quality, the database was checked for the variables it contains before the beginning of the data collection period. The collected data were checked for completeness, and the missing values were cleaned before data analysis. Missing data were checked from the patient charts. One-day orientation on the data collection tool and objective of the study was provided to the data collector and supervisor by the principal investigator.

\section{Data analysis}

All data of confirmed COVID-19 cases admitted during the study period and registered on the Microsoft excel database in the hospital were cleaned, exported, coded, and analyzed by person, place, and time by using SPSS version 22. Tables, figures, and texts were used to summarize the descriptive statics of the study. Mean and standard deviation were used for numerical data.

\section{Operational definitions}

Based on the WHO Interim Guidance for Public Health Surveillance for COVID-19 and International Guidelines for Certification and Classification[13, 14]:

Suspected case: A patient with acute respiratory illness (fever AND at least one sign/symptom of respiratory disease (e.g., cough, shortness of breath), regardless of a history of travel to or residence in a location reporting community transmission of COVID -19 disease, or regardless of having contact history 
with a confirmed or probable COVID-19 case, or Requiring hospitalization AND in the absence of an alternative diagnosis that fully explains the clinical presentation.

Confirmed case: A person with laboratory confirmation of COVID-19 infection, irrespective of clinical signs and symptoms

Death due to COVID-19: A COVID-19 death is defined for surveillance purposes as a death resulting from a clinically compatible illness in a probable or confirmed COVID-19 case unless there is a clear alternative cause of death that cannot be related to COVID-19 disease (e.g. trauma). There should be no period of complete recovery between the illness and death.

Case-fatality rate (CFR): the percentage of persons diagnosed as having a specified disease who die as a result of that disease within a given period, usually expressed as a percentage (cases per 100).

\section{Results}

\section{Baseline characteristics}

A total of 260 Real-Time PCR confirmed COVID-19 among admitted cases at Guder Hospital were included from the positive track database available in the hospital. Of these cases, $180(69.2 \%)$ were male and $90(34.6 \%)$ were found in the age group of 25-34 years. The age of cases ranges from 8 to 85 years with a mean age of 35.5 years and a standard deviation (SD) of \pm 15.0 years. Regarding their occupational statuses, the highest proportion of the cases $105(40.4 \%)$ were governmental employed and 66(25.4\%) were merchants respectively (TABLE I).

\section{Admission status}

Of the total cases, $184(70.8 \%)$ were asymptomatic, and 40(15.4\%) were mild cases i.e they developed upper respiratory tract infection (URTI) like symptoms with no evidence of pneumonia. Eighteen (6.9\%) of the admitted cases were severe, developing severe diseases including pneumonia and shortness of breath. On admission, the frequent symptoms were cough (43[16.5\%]) and shortness of breath (30[11.5\%]). Other symptoms include fatigue, headache, muscle ache, sore throat, confusion, chest pain, and loss of smell or taste. The more the old age category, the more they were symptomatic (FIGURE 1).

\section{Clinical outcomes}

Among 260 confirmed cases, 243(93.5\%) were recovered and the Case Fatality Rate (CFR) among the admitted cases during the study period was $12(4.6 \%)$. The average length of stay in the hospital was 14.3 days with SD \pm 3.6 days (TABLE 2). 
The admission of confirmed COVID-19 cases at Guder Hospital started on WHO week 20, 2020 with mean weekly admission of eight cases. The spike observed on Week 41, 2020 was the result of a mass test campaign for risk populations in the West Shewa zone (FIGURE 2).

\section{Description of COVID-19 death}

Among 12 deaths at the treatment center, more than half (58.3\%) were females. Of these deaths, eight (66.7\%) patients had known comorbidity. The mean age was 49 years (SD \pm 10.6$)$ and ranges from 35 to 75 years. The mean length of stay in ICU was 4.4 days (SD \pm 4.5$)$. Six (50\%) were on mechanical ventilators before their death. Among 12 COVID-19 deaths in the hospital, the most deadly affected age group was 45-59 years; the Age-Specific CFR was 7(17.5\%).

\section{Discussion}

This study was conducted to assess the epidemiology and outcome of confirmed COVID-19 among admitted cases to Guder Hospital as of December 31, 2020. The finding of this study revealed that adult males were more infected; children and youth below the age of 25 make up $22.7 \%$ of the total admitted cases. This result is almost similar to a study done in Ethiopia, that shows children and youth below the age of 25 make up around $30 \%$ of the total COVID-19 cases [15]. This similarity might due to school closures all over the country due to an outbreak of COVID-19 which might have prevented children from exposure to transmission sources.

According to this study, only $10 \%$ of those died were older age group (60 and above years). Of total deaths, seven (58.3\%) were females aged $45-59$ years, and eight (66.7\%) deaths had pre-existing comorbidities. Relatively few cases, 6 (2.3\%) and no death were seen among children under 15 years. This finding is different from a study conducted in Wuhan, China that showed the COVID-19 infection is more likely to affect older males with comorbidities [16]. This might be due to differences in occupational characteristics and life expectancies of the study populations.

The mean age of admitted cases was 35.5 (SD 15) years. The average length of hospitalization was 14.3 (SD 3.6) days. This finding agrees with other studies conducted among hospitalized cases in China [17]. A study conducted in Zhejiang province, China revealed that children accounted for roughly $5 \%$ of total patients with COVID19 with a mean duration of hospitalization, 14 [SD 3] days [18]. This indicates that COVID-19 affects people differently, in terms of biological sex and age which might be due to their occupational differences.

Based on all 260 admitted COVID-19 confirmed cases in Guder Hospital as of December 31, 2020, this study has found that more than a quarter (29.2\%) had one or more symptoms at admission, the commonest symptom being cough (16.5\%). These findings were different from a study done in China as of February 11, 2020, which has found that the majority (80.9\%) of infections are mild with flu-like symptoms [17] and another study done in the Oromia Regional State of Ethiopia that showed only 64 
$(0.7 \%)$ of the cases had symptoms at diagnosis[19]. These differences might be due to the immunological factor of individuals and considering mild symptoms as not a disease in the study area.

Regarding the outcome status of 260 admitted cases, $93.5 \%$ were recovered. This finding agrees with WHO data as of December 19,2020 , that revealed $1.8 \%$ of people infected with SARS-CoV-2 have a fatal outcome, while 98.2\% recovered in Ethiopia[2], however, different from other study conducted in the Oromia Region of Ethiopia that showed COVID-19 case fatality rate of $1.2 \%$ among all infected cases [19]. The difference in the current study might be due to the clinical status of the admitted cases were different from the general confirmed cases, and currently, the principle of home-based isolation and care for asymptomatic and mild cases have been implemented in Ethiopia.

The Case Fatality Rate (CFR) among the admitted cases in this study was $4.6 \%$, which was above the WHO's overall estimation of around $2 \%$, in the WHO press conference held on January 29, 2020 [16]. This difference might be due to only symptomatic cases are admitted to the treatment center in the study area and a large proportion of infected individuals who are asymptomatic isolated at their homes. Age group 45-59 years were the most affected with an age-specific case fatality rate of $17.5 \%$ in this study. This might be due to lower life expectancies in Ethiopia.

Strength: This study added baseline information to design and implement mitigation strategies.

Limitation: This study encountered data incompleteness and missing important independent variables (travel history, contact history, BMI, etc) to conduct an analytical study and establish a cause-effect relationship for the infection.

\section{Conclusions}

Overall, the finding of this study revealed that COVID-19 infection is more likely to affect adult males among admitted cases in the early ten months of infection. The disease remains largely asymptomatic and they recovered after 14 days mean time hospitalization. Even though people of all ages can be infected by COVID-19, male adults (15-59 years), and government-employed peoples appear to be more vulnerable to the infection with the virus among admitted cases. In general, COVID-19 infection is more common in males and death is more likely in females with comorbidities. Therefore, mitigation strategies should be sustainably implemented among admitted cases as well as in the general community to avert morbidity and mortality due to COVID-19 infection especially targeting the most vulnerable group.

\section{What Is Already Know On This Topic And What This Study Adds}

No evidence was available about the epidemiological, clinical features, and outcome of admitted patients in the study area. This study aimed to provide insight into the epidemiological characteristics and outcome of confirmed COVID-19 infection among admitted cases at Guder Hospital, Oromia Regional State, Ethiopia. 


\section{Declarations}

\section{Competing interests}

The authors declare that they have no competing interests.

\section{Authors' Contributions}

TS developed the proposal, conducted the analysis, wrote the manuscript, and had full access to all the data in the study, and had final responsibility for the decision to submit for publication. TK provided general guidance, participated in reviewing the proposal, analyzing and developing the final study document. MT involved in data cleaning and reviewing the manuscript. All authors read and approved the final manuscript.

\section{Acknowledgements}

The authors acknowledge Oromia Regional Health Bureau, West Shewa Zonal Health Office, and Guder Hospital for their cooperation. The authors declared no fund has been received.

\section{Ethics approval and consent for publication}

Letter of ethical clearance was obtained from the Ethics Committee of Oromia Regional Health Bureau, Public Health Emergency Management and Health Research Directorate, and officially submitted to West Shewa Zone Health Department and Guder Hospital. The ethics committee approved the procedure for data collection by replacing the name of patients on the database with codes for confidentiality reasons. The Ethics committee approved that the finding of the study could publish since identifying images or clinical details of participants that compromise anonymity was not applicable.

\section{Availability of data and materials}

The data supporting our findings are found at, kept in confidentiality, and stored at the corresponding author both in soft copy.

\section{References}

1. WHO, Clinical management of COVID-19 interim guidance. May 2020 (WHO/2019nCoV/clinical/2020.5).

2. WHO, www.worldometer.info_ https://www.worldometers.info/coronavirus/\#countries accessed on December 19, 2020.

3. WHO, COVID-19 as a Public Health Emergency of International Concern (PHEIC) under the International Health Regulation (IHR) Fact Sheet 15A, May 2020.

4. WHO, Strategic partnership for International Health Regulations (2005) and health security (SPH). Timor-Leste boosts health security by aligning NAPHS with pandemic influenza preparedness 
(https://extranet. who. int/sph/news/timor-leste-boosts-health-security-aligning-naphs-pandemicinfluenza-preparedness, accessed 12 January 2020).

5. WHO, www.worldometer.info_https://www.worldometers.info/coronavirus/\#countries accessed on December 31, 2020.

6. EPHI, Ethiopia confirmed the first case of COVID-19, 2020. Press Release, EPHI

7. Coronavirus - Ethiopia: https://www.africanews.com/2020/12/31/coronavirus-ethiopia-covid-19reported-cases-in-ethiopia-31-december-2020//, in Africanews.

8. FMOH., Special bulletin $22^{\text {nd }}$ annual review meeting November, 2020.

9. WHO, Coronavirus Symptoms (COVID-19)-Worldometerhttps://www.worldometers.info/coronavirus/coronavirus-symptoms/ accessed on December 19, 2020.

10. EPHI, National Public Health Emergency Operating Center COVID-19 Emergency Preparedness and Response. 2020.

11. Management, E.P.H.I.C.f.P.H.E., Discharge criteria for Novel Corona virus (nCoV 2019) cases admitted to Treatment center. 2020.

12. West Shewa Zonal Health Office and T.f.P.H.E. Management, West Shewa Zonal Health Office and Oromia Regional Health Bureau COVID-19 Report. 2020..

13. WHO, Public health surveillance for COVID-19: Interim guidance. 7 August 2020.

14. Watare, S.H., et al., The First Hundred Days of Novel Corona-virus Disease 2019 in Ethiopia, 13 March to 21 June 2020: Retrospective Analysis. 2020.

15. Bender, L., Interim Guidance for COVID-19 Prevention and Control in Schools. UNICEF, 2020.

16. Chen, N., et al., Epidemiological and clinical characteristics of 99 cases of 2019 novel coronavirus pneumonia in Wuhan, China: a descriptive study. The lancet, 2020. 395(10223): p. 507-513.

17. Wang, D., et al., Clinical characteristics of 138 hospitalized patients with 2019 novel coronavirusinfected pneumonia in Wuhan, China. Jama, 2020. 323(11): p. 1061-1069.

18. Qiu, H., et al., Clinical and epidemiological features of 36 children with coronavirus disease 2019 (COVID-19) in Zhejiang, China: an observational cohort study. The Lancet Infectious Diseases, 2020. 20(6): p. 689-696.

19. Gudina, E.K., et al., Epidemiology of COVID-19 Outbreak in Oromia Region of Ethiopia: The First Six Months Surveillance-13 March 2020 to 13 September 2020. 2020.

\section{Tables}

Table 1: Demographics, baseline characteristics, and admission status of confirmed COVID-19 among admitted cases at Guder Hospital, Oromia Regional State, Ethiopia, January 2021 


\begin{tabular}{|c|c|c|c|}
\hline Characteristic & Categories & Frequency & Percent (\%) \\
\hline \multirow[t]{6}{*}{ Age category } & $5-14$ & 6 & $2.3 \%$ \\
\hline & $15-24$ & 53 & $20.4 \%$ \\
\hline & $25-34$ & 90 & $34.6 \%$ \\
\hline & $35-44$ & 45 & $17.3 \%$ \\
\hline & $45-59$ & 40 & $15.4 \%$ \\
\hline & $>=60$ & 26 & $10.0 \%$ \\
\hline \multirow[t]{2}{*}{ Sex } & Male & 180 & $69.2 \%$ \\
\hline & Female & 80 & $30.8 \%$ \\
\hline \multirow[t]{9}{*}{ Occupation } & Government employed & 105 & $40.4 \%$ \\
\hline & Merchant & 66 & $25.4 \%$ \\
\hline & Student & 25 & $9.6 \%$ \\
\hline & Prisoners & 18 & $6.9 \%$ \\
\hline & Housewife & 11 & $4.2 \%$ \\
\hline & Road construction & 11 & $4.2 \%$ \\
\hline & Blank & 10 & $3.8 \%$ \\
\hline & Farmer & 7 & $2.7 \%$ \\
\hline & Self-employed & 7 & $2.7 \%$ \\
\hline \multirow{10}{*}{ Residence } & West Shewa & 194 & $74.6 \%$ \\
\hline & Ambo Town & 34 & $13.1 \%$ \\
\hline & Horo Guduru Wollega & 14 & $5.4 \%$ \\
\hline & Finfine Special & 7 & $2.7 \%$ \\
\hline & Burayu Town & 5 & $1.9 \%$ \\
\hline & Gambella & 2 & $0.8 \%$ \\
\hline & Finfine & 1 & $0.4 \%$ \\
\hline & Gelan Town & 1 & $0.4 \%$ \\
\hline & Jimma & 1 & $0.4 \%$ \\
\hline & Tigray & 1 & $0.4 \%$ \\
\hline \multirow[t]{8}{*}{ Month of admission } & May & 5 & $1.9 \%$ \\
\hline & June & 16 & $6.2 \%$ \\
\hline & July & 43 & $16.5 \%$ \\
\hline & August & 121 & $46.5 \%$ \\
\hline & September & 2 & $0.8 \%$ \\
\hline & October & 39 & $15.0 \%$ \\
\hline & November & 19 & $7.3 \%$ \\
\hline & December & 15 & $5.8 \%$ \\
\hline \multirow[t]{5}{*}{ Status at admission } & No symptom & 184 & $70.8 \%$ \\
\hline & Mild & 40 & $15.4 \%$ \\
\hline & Moderate & 14 & $5.4 \%$ \\
\hline & Severe & 18 & $6.9 \%$ \\
\hline & Critical (ICU) & 4 & $1.5 \%$ \\
\hline \multirow[t]{4}{*}{ Length of stay } & $<2$ days & 5 & $1.9 \%$ \\
\hline & 2-7 days & 14 & $5.4 \%$ \\
\hline & 8-14 days & 38 & $14.6 \%$ \\
\hline & $>=15$ days & 203 & $78.1 \%$ \\
\hline
\end{tabular}

Table 2: Clinical outcomes of confirmed COVID-19 among admitted cases at Guder Hospital by demographic characteristics and admission statuses, Oromia Regional State, Ethiopia, January 2021 
Died Lost Recovered Referred Total Percent

\begin{tabular}{|c|c|c|c|c|c|c|c|}
\hline \multirow{6}{*}{$\begin{array}{l}\text { Status } \\
\text { admission }\end{array}$} & $\begin{array}{lll}\text { at } & \text { No sign } \\
\text { svmptom }\end{array}$ & 1 & 1 & 180 & 2 & 184 & $70.8 \%$ \\
\hline & Mild & 2 & 0 & 38 & 0 & 40 & $15.4 \%$ \\
\hline & Moderate & 0 & 1 & 13 & 0 & 14 & $5.4 \%$ \\
\hline & Severe & 5 & 0 & 12 & 1 & 18 & $6.9 \%$ \\
\hline & Critical & 4 & 0 & 0 & 0 & 4 & $1.5 \%$ \\
\hline & Total & 12 & 2 & 243 & 3 & 260 & $100.0 \%$ \\
\hline \multirow[t]{7}{*}{ Age category } & $5-14$ & 0 & 0 & 6 & 0 & 6 & $2.3 \%$ \\
\hline & $15-24$ & 0 & 0 & 52 & 1 & 53 & $20.4 \%$ \\
\hline & $25-34$ & 0 & 1 & 88 & 1 & 90 & $34.6 \%$ \\
\hline & $35-44$ & 4 & 0 & 40 & 1 & 45 & $17.3 \%$ \\
\hline & $45-59$ & 7 & 1 & 32 & 0 & 40 & $15.4 \%$ \\
\hline & $>=60$ & 1 & 0 & 25 & 0 & 26 & $10.0 \%$ \\
\hline & Total & 12 & 2 & 243 & 3 & 260 & $100.0 \%$ \\
\hline \multirow[t]{3}{*}{ Sex } & Female & 7 & 0 & 73 & 0 & 80 & $30.8 \%$ \\
\hline & Male & 5 & 2 & 170 & 3 & 180 & $69.2 \%$ \\
\hline & Total & 12 & 2 & 243 & 3 & 260 & $100.0 \%$ \\
\hline \multirow[t]{10}{*}{ Occupation } & $\begin{array}{l}\text { Government } \\
\text { employed }\end{array}$ & 1 & 0 & 103 & 1 & 105 & $40.4 \%$ \\
\hline & Merchant & 3 & 0 & 62 & 1 & 66 & $25.4 \%$ \\
\hline & Student & 1 & 0 & 24 & 0 & 25 & $9.6 \%$ \\
\hline & Prisoners & 0 & 1 & 17 & 0 & 18 & $6.9 \%$ \\
\hline & Road Construction & 0 & 0 & 11 & 0 & 11 & $4.2 \%$ \\
\hline & Housewife & 3 & 0 & 8 & 0 & 11 & $4.2 \%$ \\
\hline & Blank & 4 & 1 & 4 & 1 & 10 & $3.8 \%$ \\
\hline & Self-employed & 0 & 0 & 7 & 0 & 7 & $2.7 \%$ \\
\hline & Farmer & 0 & 0 & 7 & 0 & 7 & $2.7 \%$ \\
\hline & Total & 12 & 2 & 243 & 3 & 260 & $100.0 \%$ \\
\hline
\end{tabular}

\section{Figures}




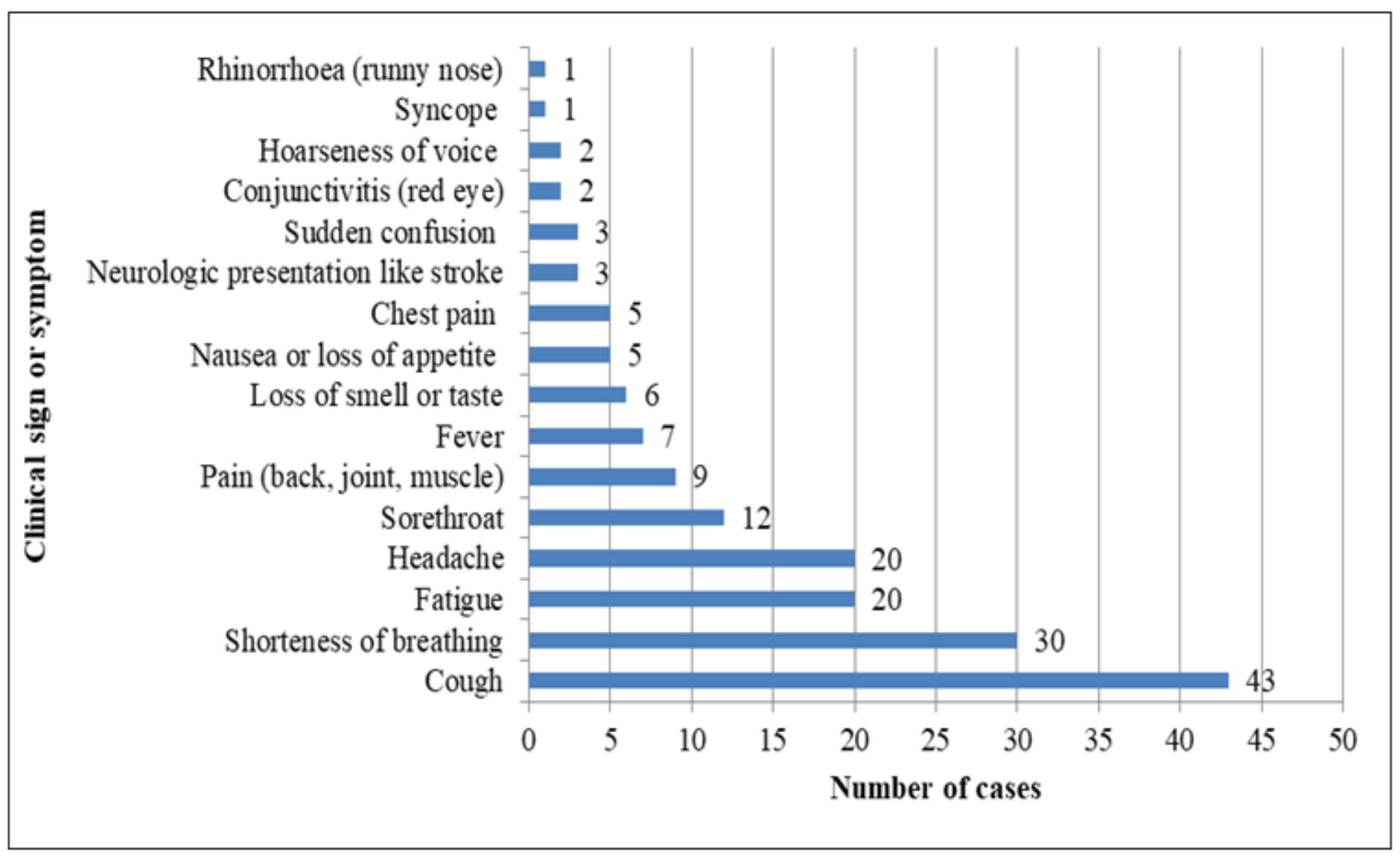

Figure 1

Clinical presentation of confirmed COVID-19 among admitted cases at Guder Hospital, January 2021

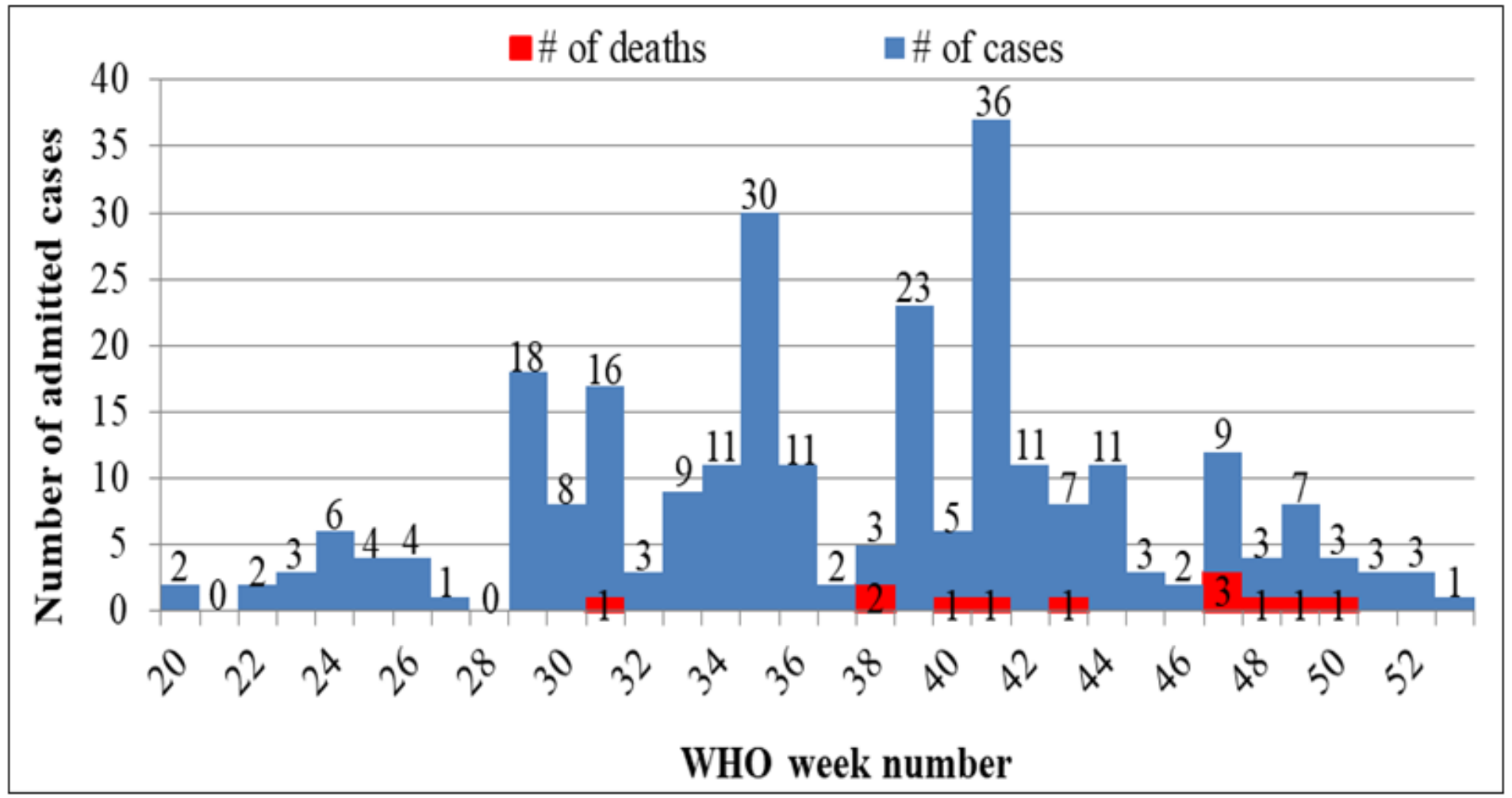


Figure 2

Epidemic curve of confirmed COVID-19 among admitted cases at Guder Hospital, Oromia Regional State, WHO week 20 to 53,2020 